\title{
Tight glycemic control in critically ill pediatric patients: a meta-analysis and systematic review of randomized controlled trials
}

\author{
Yiyang Zhao ${ }^{1}$, Yang $\mathrm{Wu}^{1}$ and Bo Xiang ${ }^{1}$
}

BACKGROUND: There still are controversies in the impact of tight glycemic control (TGC) in critically ill children. The aim of this study was to assess the benefits and risks of TGC compared with conventional glycemic control (CGC) in critically ill pediatric patients admitted to the pediatric intensive care unit (PICU) by using the data retrieved from randomized controlled trials (RCTS).

METHODS: EMBASE, CNKI, PubMed, and the Cochrane Database were searched for RCTs comparing TGC with CGC in critically ill children in PICU.

RESULTS: The meta-analysis included 5 RCTs representing 3,933 patients that compared TGC with CGC. Our result revealed that TGC did not reduce 30-day mortality rates (odds ratio (OR) 0.99, 95\% confidence interval $(\mathrm{Cl}) 0.74-1.32, P=0.95$ ) and was not associated with decreasing health careassociated infections (OR 0.80, 95\% Cl 0.64-1.00, $P=0.05$ ) compared with CGC, but significantly increased the incidence of hypoglycemia (OR 6.37, 95\% Cl 4.41-9.21, P<0.001).

CONCLUSION: Tight glycemic control was not associated with reducing 30-day mortality rates and acquired infections compared with CGC in critically ill children. Significant increase of incidence of hypoglycemia was revealed in TGC group. The conclusion should be interpreted with caution for the methodological heterogeneity among trials.

$\mathbf{H}$ yperglycemia is a common phenomenon in critically ill patients (1-5). Persistent hyperglycemia state during critical ill may induce increased oxidative damage, proinflammatory response, and tissue impairment (6-8). Several studies have showed hyperglycemia is associated with poor prognosis in critical patients $(1,2,9-15)$. Considering the potential benefits of tight glycemic control in critically ill patients, several trials of TGC in adult intensive care units had been carried out and some of them showed advantages of TGC in reducing morbidity or mortality $(16,17)$, while other trials did not (18-20). However, two meta-analyses were not able to show a benefit $(21,22)$. By now there were a few randomized control trials (RCTs) comparing TGC with CGC involving critically ill pediatric patients with different outcomes that had been published (23-27). Because of the controversies of tight glycemic controls in critically ill children, we performed this meta-analysis and systematic review to evaluate the benefits and adverse effects of TGC compared with CGC.

\section{Methods}

\section{Eligibility Criteria}

We included studies fulfilling the following criteria in the present systemic review: RCTs, studies that evaluated clinical outcomes in critically ill children who had received tight glycemic control with insulin therapy. There was no limit on publication status or language. The primary outcome of analysis was the 30-day mortality rate of the patients. If 30-day mortality was not reported, we used 28-day mortality or ICU mortality as reported. The secondary outcomes were the rates of occurrence of health care-associated infections during ICU stay and incidence of hypoglycemia. Hypoglycemia is defined as $<40 \mathrm{mg} / \mathrm{dl}$; in case this is missing, the incidence of hypoglycemia with a cutoff closest to $40 \mathrm{mg} / \mathrm{dl}$ is used. The incidence of hypoglycemia was defined as the proportion of patients experiencing at least one single episode of hypoglycemia during ICU stay. Exclusion criteria were: non-RCT studies, non-human subjects, impossible to extract data from the articles; and overlapping authors, institutes, or patients in the published literatures. We also excluded RCTs related with glycemic control in low-birth-weight neonates and adults.

\section{Search Strategy and Selection of Studies}

We systemically searched MEDLINE (PubMed as the search engine), EMBASE, Ovid, ISI Web of Science and the Cochrane Library from 1 January 1964 to 12 May 2017, by two independent reviewers (Yiyang Zhao and Yang $\mathrm{Wu}$ ). Search terms were selected as: hyperglycemia, glycemic control, insulin, glucose control, hypoglycemia, pediatric and children, critical and intensive care. For PubMed, the Medical Subject Heading (MeSH) and textword search were adopted. For EMBASE, Ovid, and the Cochrane Library, searches using key word Headings were performed (http://links.lww.com/MPG/A418). References of previous reviews were also searched for related studies. Two reviewers (Yiyang Zhao and Yang $\mathrm{Wu}$ ) independently evaluated all the retrieved articles using the reported eligibility criteria. In case of disagreement, a consensual decision was made. All relevant studies were first scanned with title and abstracts. Selected full articles were then scanned and assessed. We also searched references, related book chapters, and conference presentations. Studies that met selection criteria were finally included and analyzed.

\section{Data Extraction}

Two previous reviewers had extracted data from included RCTs into RevMan 5.0 software independently. These data included

\footnotetext{
'Department of Pediatric Surgery, Sichuan University West China Hospital, Chengdu, Sichuan, China. Correspondence: Bo Xiang (xb_scu.edu@hotmail.com) The first two authors contributed equally to this work. 
characteristics of studies, patient demographic information, patterns of interventions, clinical outcomes, and complications. The corresponding author would be contacted to get supplementary data when there is missing information or inaccuracies.

\section{Quality Assessment}

Methodological quality of included studies was assessed by using the Jadad scale including a maximum of 7 points to randomization, double blinding, dropouts and withdrawals, and allocation concealment. Two authors examined the studies independently. Same consensus process mentioned above was used to resolve disagreements.

\section{Statistical Analysis}

Meta-analysis was performed in line with recommendations from the Cochrane Collaboration and the Quality of Reporting of metaanalyses guidelines $(28,29)$. Statistical analyses were carried out using Review Manager version 5.0 (Cochrane Collaboration, Oxford, UK). Dichotomous variables were analyzed with the Mantel-Haenszel statistical method using odds ratio (OR) as the summary statistic, and both were reported with 95\% confidence interval (CI). Clinical heterogeneity was tested by means of the $I^{2}$ value and a value exceeding $50 \%$ was considered to represent a significant difference. A random-effects model was used to report the results of heterogeneous data; otherwise, a fixed-effects model was used. The OR was considered to be statistically significant at $P<0.05$ if the $95 \%$ CI did not include the value " 1 ." Funnel plots were constructed to detect and assess publication bias and any association between treatment estimates and sample sizes.

\section{RESULTS}

\section{Evaluations of Qualities of Trials}

Figure 1 illustrated the detailed information of literature search and PRISMA flowchart. Initial search yielded 58 relevant articles. No additional studies had been identified by

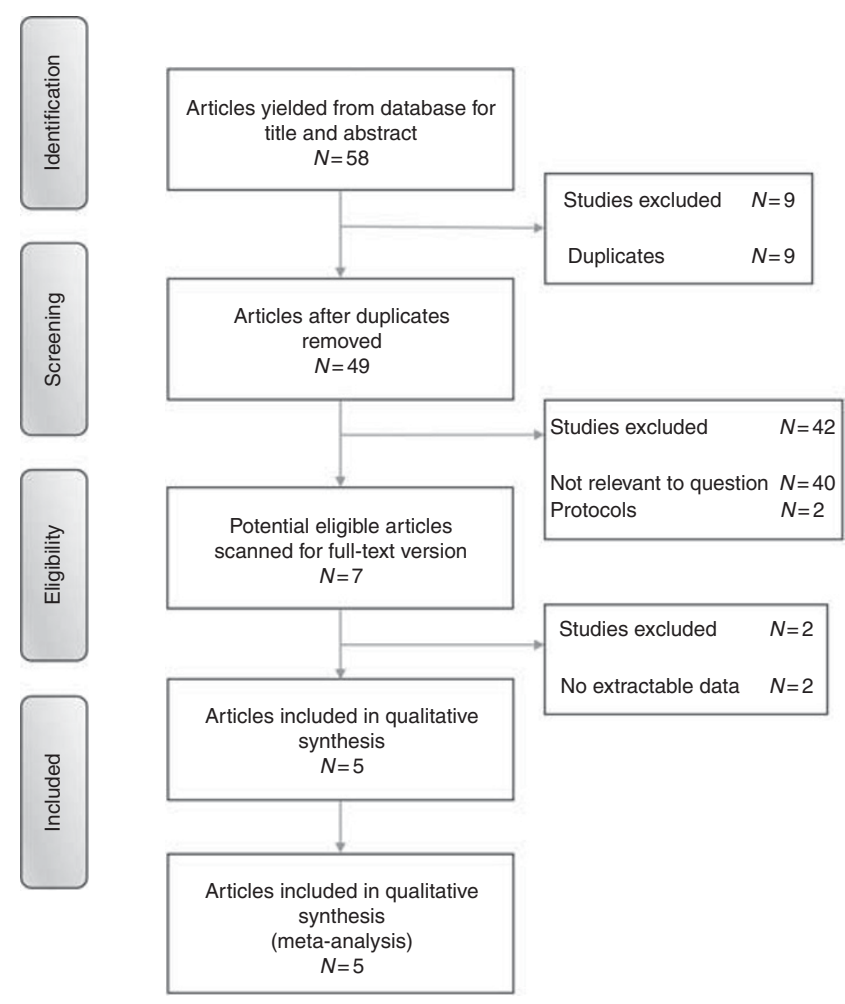

Figure 1. PRISMA flow diagram of literature search.

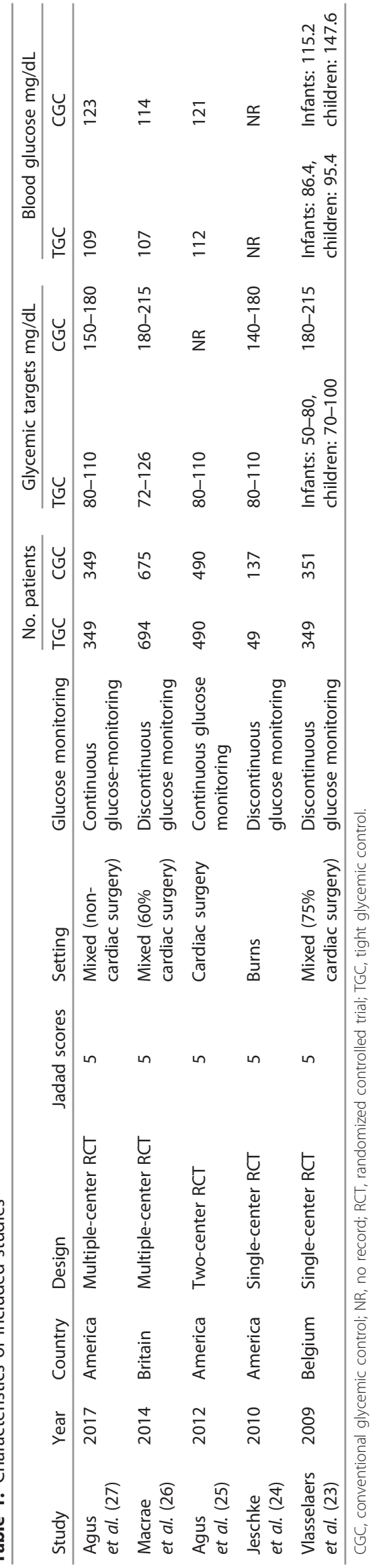




\section{Systematic Review | Zhao et al.}

cross-referencing the bibliographies of relevant articles. We checked the title and abstract of each article and seven of the total included studies had been selected for further reviews. Finally, a total of five RCTs had been included in the metaanalysis. All the included studies were classified as high quality (Jadad score $\geqslant 4$, Table 1 ).

\section{Characteristics and Baseline Information of Included Trials}

A total of 3,933 patients had been included in this study. The number of patients in each trial varied from 186 to 1,369 . These trials were performed in pediatric patients (containing severely burned, cardiac surgical, mixed critically ill). It should be noted that these studies had different glycemic targets and achieved different glucose levels in TGC and CGC groups. Table 1 showed characteristics and baseline information of included trials.

\section{Study Outcomes}

The primary outcome of this study was the 30-day mortality rate of admission of the patients. Three trials reported mortality rates within 30 days $(23,25,26)$, the HALF-PINT trial reported 28-day mortality (27), and Jeschke trial only reported ICU mortality (24). If 30-day mortality was not reported, we used 28-day mortality or ICU mortality as reported. Analysis with pooled data showed that tight glycemic controls in critically ill children had not reduced mortality rates (OR $0.99,95 \%$ CI $0.74-1.32, \quad P=0.95$ ) (Figure 2a). The secondary outcome was infection rate of patients between TGC and CGC groups. It was illustrated that TGC may be associated with decrease in acquired infection, but there was no statistical difference (OR 0.80, 95\% CI 0.64$1.00, P=0.05$; Figure 2b). Hypoglycemia as a major complication of glucose control was reported in all of the five studies. Four trials defined severe hypoglycemia as blood glucose level below $40 \mathrm{mg} / \mathrm{dl}$, except trial by Macrae et al. (26) that defined severe hypoglycemia as blood glucose level $<36 \mathrm{mg} / \mathrm{dl}$. Our study showed that TCG in critically ill children increased the incidence of hypoglycemia (OR 6.37, 95\% CI 4.41-9.21, $P<0.001$; Figure 2c).

\section{Publication Bias}

Funnel plot of this study based on mortality was shown in Figure 3. All of the included studies lay inside the limits of the 95\% CI and distributed evenly about the vertical, showing no evidence of publication bias.

\section{DISCUSSION}

The present meta-analysis showed that there currently were no obvious benefits of TGC compared with CGC in critically ill children. Tight glycemic control did not reduce mortality rates (OR 0.99, 95\% CI 0.74-1.32, $P=0.95$ ) and was not associated with decreasing infection rates (OR 0.80, 95\% CI $0.64-1.00, P=0.05)$. Significantly increased incidence of hypoglycemia was revealed in the TGC groups (OR 6.37, 95\% CI 4.41-9.21, $P<0.001)$. Although the trial by Vlasselaers et al. (23) showed that TGC had benefits of reducing lengths of PICU stay and mortality in critical children (75\% patients receiving cardiac surgery). Jeschke et al. (24) showed advantages of TGC compared with CGC on improving organ functions and alleviating post-burn insulin resistance in severe burned children, but found no significant differences in mortality rates between TGC and CGC groups. The study by Macrae et al. (26) involving a mixed population of critically ill children was unable to show any statistically positive effects on major clinical outcomes with TCG. But in the subgroup of non-cardiac surgical patients, the mean 12month cost was significantly lower in the TGC group than in the CGC group. The other two studies did not show benefits on mortality or lengths of PICU stay $(25,27)$.

The study by Vlasselaers et al. (23) indicated that mortality was lower in TGC group possibly due to the prevention of infection and pulmonary damage, and protective effects of heart. They also stated that their sample size was not large enough to confirm these benefits. Our study analyzed 3,933 pediatric patients and intended to expand the trial volume. Both in vitro and in vivo studies revealed that insulin therapy had positive effects of improving organ functions and alleviating inflammatory responses (30-32). Although tight glycemic control may bring clinical benefits in theory, the pooled analysis of present study did not show obvious survival benefits of TGC compared with CGC in critically ill children. The heterogeneity of critical degrees of patients may partially explain the result. Several trials had small difference in achieved blood glucose levels. This may explain why the mortality rate was not significantly different. If there were benefits with a minor difference in blood glucose levels, which would be likely very small.

Two of the included studies revealed benefits of reducing infection rates with TGC $(23,24)$, another two studies showed no significance in infection rates between TGC and CGC groups $(25,26)$. The recent trial by Agus et al. (27) indicated that TGC group had higher rates of health care-associated infections compared with CGC group. Studies had showed that insulin induced expression of endothelial NO synthase, suppressed intracellular adhesion molecule-1, monocyte chemoattractant protein-1 expression, and played antiinflammatory and antioxidant roles (33-35). Although our study did not show benefit of reducing infection rates with TGC, it probably was due to the complicated condition of critically ill pediatric patients. For example, insulin therapy may exert more obvious anti-inflammatory effect in obese or diabetic patients (36-38). Discrepancy of susceptibility to infection and different antibiotic regimens of trials may also contribute to this result.

Hypoglycemia is a major complication of TGC and associated with clinical outcomes particularly in pediatric patients $(19,39,40)$. Although the SPECS trial by Agus et al. showed a low rate of severe hypoglycemia in TGC group compared with CGC group (3\% vs. $1 \%$ ), all of the included studies indicated that TGC can significantly increase the incidence of hypoglycemia despite the use of insulin-dosing algorithm and continuous subcutaneous glucose monitoring. 


\section{Tight glycemic control in PICU $\mid$ Systematic Review}

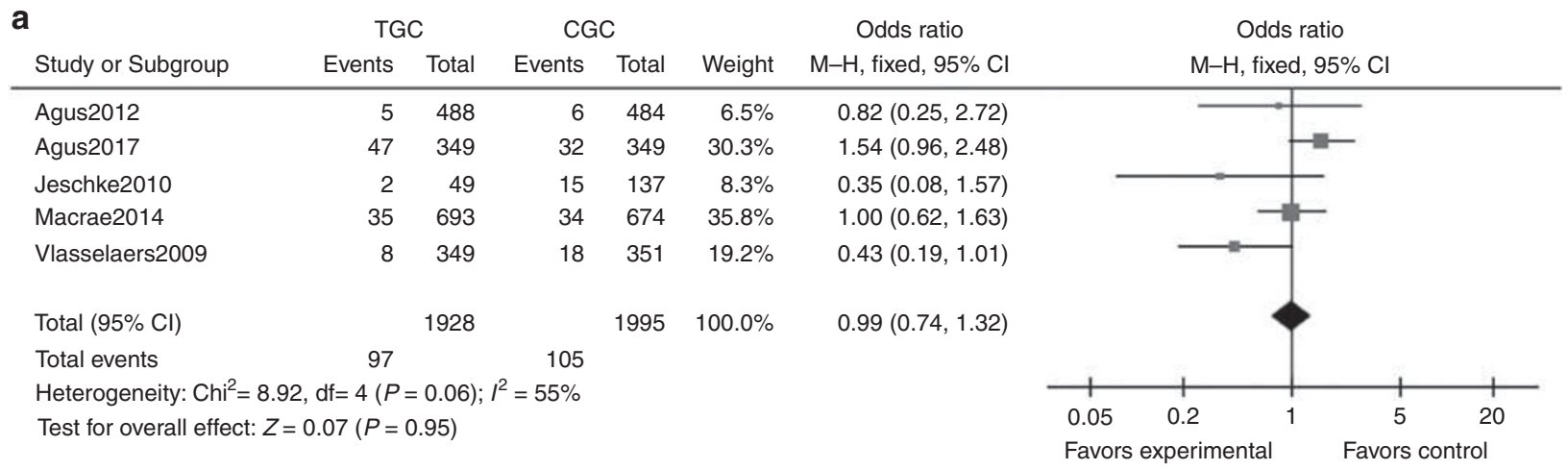

\begin{tabular}{|c|c|c|c|c|c|c|}
\hline \multirow{2}{*}{ Study or Subgroup } & \multicolumn{2}{|c|}{ TGC } & \multicolumn{2}{|c|}{ CGC } & \multirow[b]{2}{*}{ Weight } & \multirow{2}{*}{$\begin{array}{l}\text { Odds ratio } \\
\text { - fixed, } 95 \% \mathrm{Cl}\end{array}$} \\
\hline & Events & Total & Events & Total & & \\
\hline Agus2012 & 24 & 490 & 24 & 490 & $13.1 \%$ & $1.00(0.56,1.79)$ \\
\hline Agus2017 & 12 & 349 & 4 & 349 & $2.2 \%$ & $3.07(0.98,9.62)$ \\
\hline Jeschke2010 & 4 & 49 & 31 & 137 & $8.6 \%$ & $0.30(0.10,0.91)$ \\
\hline Macrae2014 & 38 & 694 & 43 & 675 & $23.7 \%$ & $0.85(0.54,1.34)$ \\
\hline Vlasselaers2009 & 102 & 349 & 129 & 351 & $52.3 \%$ & $0.71(0.52,0.98)$ \\
\hline Total $(95 \% \mathrm{Cl})$ & & 1931 & & 2002 & $100.0 \%$ & $0.80(0.64,1.00)$ \\
\hline Total events & 180 & & 231 & & & \\
\hline
\end{tabular}

Heterogeneity: $\mathrm{Chi}^{2}=9.50, \mathrm{df}=4(P=0.05) ; I^{2}=58 \%$

Test for overall effect: $Z=1.97(P=0.05)$

\begin{tabular}{lrrrrr} 
C & \multicolumn{4}{c}{ TGC } & \multicolumn{1}{c}{ CGC } \\
Study or Subgroup & Events & Total & Events & Total & Weight \\
\hline Agus2012 & 16 & 490 & 5 & 490 & $16.5 \%$ \\
Agus2017 & 18 & 349 & 7 & 349 & $22.7 \%$ \\
Jeschke2010 & 13 & 49 & 12 & 137 & $15.9 \%$ \\
Macrae2014 & 51 & 694 & 10 & 675 & $32.1 \%$ \\
Vlasselaers2009 & 87 & 349 & 5 & 351 & $12.8 \%$ \\
& & & & & \\
Total $(95 \%$ Cl) & 185 & & 39 & \\
Total events & 1931 & & $100.0 \%$ \\
Heterogeneity: Chi $^{2}=14.66, \mathrm{df}=4(P=0.005) ; I^{2}=73 \%$ \\
Test for overall effect: $Z=9.88(P<0.00001)$
\end{tabular}

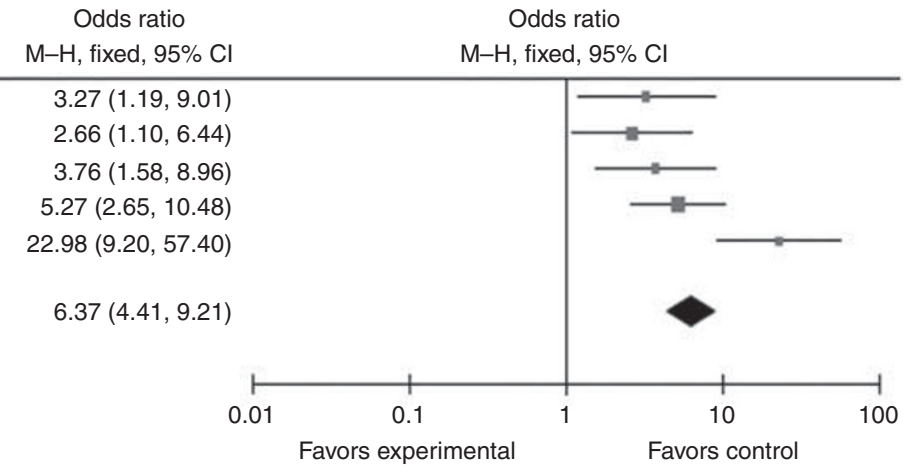

Figure 2. Forest plots of pool data analysis, comparing TGC with CGC in critically ill pediatric patients. (a) Forest plot of 30-day mortality. (b) Forest plot of health care-associated infections. (c) Forest plot of hypoglycemia (defined as blood glucose concentration $\leqslant 40 \mathrm{mg} / \mathrm{dl}(\leqslant 2.2 \mathrm{mmol} / \mathrm{l})$ ). CGC, conventional glycemic control; df, degrees of freedom; M-H, Mantel-Haenszel; SD, standard deviation; TGC, tight glycemic control.

Partly, different designs of trials may influence the incidence of hypoglycemia. Vlasselaers trial used lower target in the intervention group, had relatively higher risk of hypoglycemia (23). Hypoglycemia may impact brain and physical development in pediatric patients especially in neonates $(41,42)$. Several studies revealed that hyperglycemia and glucose fluctuation or rapid correction of hypoglycemia also may cause neurosensory impairment (43-45). The follow-up study by Vlasselaers et al. evaluated neurodevelopmental outcomes in the critically ill children who enrolled in the randomized trial of TGC vs. CGC 4 years later $(43,46)$. The result revealed that TGC with high rates of hypoglycemia did not impact neurodevelopment, possibly have benefits on neurodevelopment $(43,46)$. Sadhwani et al. assessed the impact of TGC and hypoglycemia on neurodevelopmental outcomes in patients included in the study by Agus et al. (25) when they were 1 year old (47). The study showed that TGC did not impact neurodevelopment compared with CGC, while moderate to severe hypoglycemia may be associated with poorer neurodevelopmental outcomes at 1 year of age (47). Considering the potential risk of hypoglycemia, long-term follow-up to assess the association of tight glycemic control and hypoglycemia with neurodevelopmental outcomes is needed.

The present study had limitations. The data of studies had heterogeneity. Heterogeneity of included population existed among trials. Different types of patients may have dissimilar critical degrees or clinical outcomes, we failed to perform subgroup analysis because of too many missing data. The observation periods of mortality rates were not exactly the same. If we exclude mortality of Jeschke trial (24) that 


\section{Systematic Review | zhao et al.}

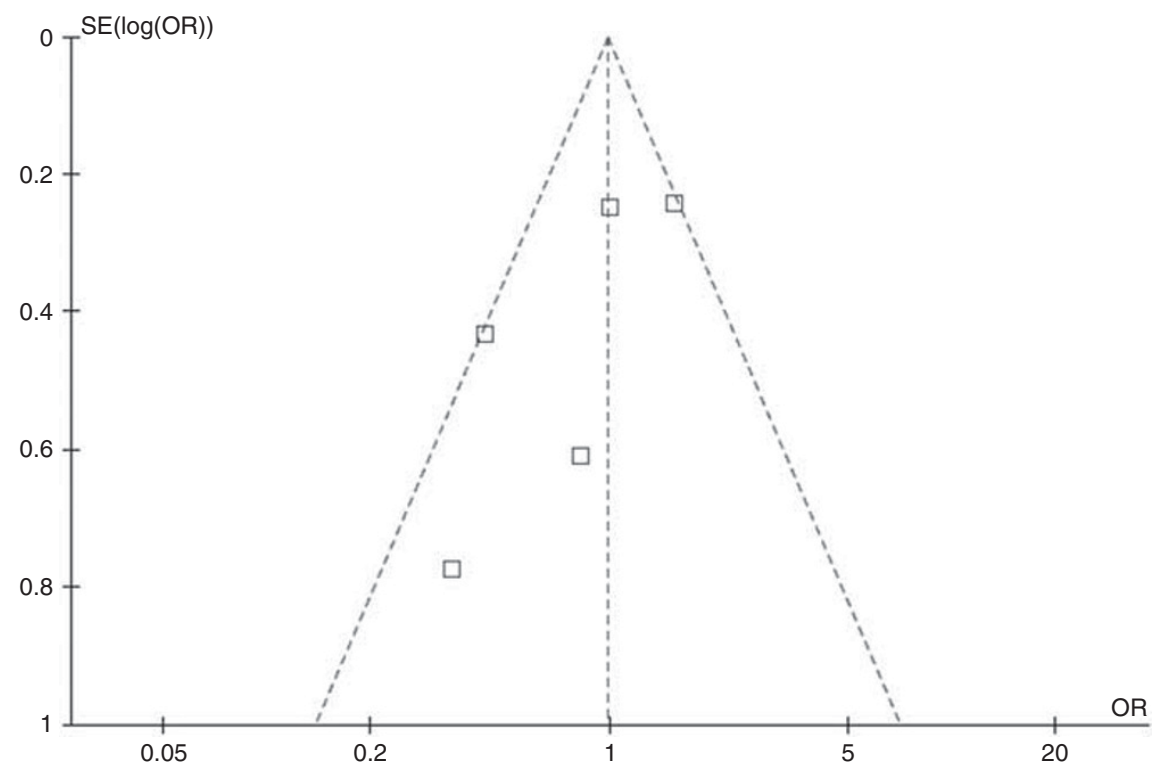

Figure 3. Funnel plot of mortality, showing no publication bias.

reported ICU mortality, there still was no statistical difference in mortality rates (OR $0.95,95 \%$ CI $0.57-1.58, P=0.84$ ). Besides, the trials did not reported incidence of health careassociated infections in a uniform way. This meta-analysis probably underestimated the incidence of acquired infections. These trials had different antibiotic regimens that also may have an impact on incidence of infections. The glycemic targets and glycemic control strategies varied among trials, the achieved glucose levels of each trial also differed.

\section{Conclusion}

This meta-analysis was not able to demonstrate any superiority of tight glycemic control compared with conventional glycemic control in critically ill children. Besides, tight glycemic control can significantly increase the incidence of hypoglycemia. In view of the heterogeneity of the included studies, the conclusion of this meta-analysis should be interpreted with caution.

Disclosure: The authors declare no conflict of interest.

\section{REFERENCES}

1. Capes SE, Hunt D, Malmberg K, Gerstein HC. Stress hyperglycaemia and increased risk of death after myocardial infarction in patients with and without diabetes: a systematic overview. Lancet 2000;355:773-8.

2. Capes SE, Hunt D, Malmberg K, Pathak P, Gerstein HC. Stress hyperglycemia and prognosis of stroke in nondiabetic and diabetic patients: a systematic overview. Stroke 2001;32:2426-32.

3. Faustino EV, Apkon M. Persistent hyperglycemia in critically ill children. J Pediatr 2005;146:30-4.

4. Yates AR, Dyke PC 2nd, Taeed R, et al. Hyperglycemia is a marker for poor outcome in the postoperative pediatric cardiac patient. Pediatr Crit Care Med 2006;7:351-5.

5. Yung M, Wilkins B, Norton L, et al. Glucose control, organ failure, and mortality in pediatric intensive care. Pediatr Crit Care Med 2008;9: $147-52$.
6. Dufour S, Lebon V, Shulman GI, Petersen KF. Regulation of net hepatic glycogenolysis and gluconeogenesis by epinephrine in humans. Am J Physiol Endocrinol Metab 2009;297:E231-5.

7. Kulp GA, Herndon DN, Lee JO, Suman OE, Jeschke MG. Extent and magnitude of catecholamine surge in pediatric burned patients. Shock 2010;33:369-74.

8. Srinivasan V. Stress hyperglycemia in pediatric critical illness: the intensive care unit adds to the stress!. J Diab Sci Technol 2012;6:37-47.

9. McCowen KC, Malhotra A, Bistrian BR. Stress-induced hyperglycemia. Crit Care Clin 2001;17:107-24.

10. Falciglia $\mathrm{M}$. Causes and consequences of hyperglycemia in critical illness. Curr Opin Clin Nutr Metab Care 2007;10:498-503.

11. Wintergerst KA, Buckingham B, Gandrud L, Wong BJ, Kache S, Wilson DM. Association of hypoglycemia, hyperglycemia, and glucose variability with morbidity and death in the pediatric intensive care unit. Pediatrics 2006;118:173-9.

12. Srinivasan V, Spinella PC, Drott HR, Roth CL, Helfaer MA, Nadkarni V. Association of timing, duration, and intensity of hyperglycemia with intensive care unit mortality in critically ill children. Pediatr Crit Care Med 2004:5:329-36.

13. Branco RG, Garcia PC, Piva JP, Casartelli CH, Seibel V, Tasker RC. Glucose level and risk of mortality in pediatric septic shock. Pediatr Crit Care Med 2005;6:470-2.

14. Piper HG, Alexander JL, Shukla A, et al. Real-time continuous glucose monitoring in pediatric patients during and after cardiac surgery. Pediatrics 2006;118:1176-84.

15. Falcao G, Ulate K, Kouzekanani K, Bielefeld MR, Morales JM, Rotta AT. Impact of postoperative hyperglycemia following surgical repair of congenital cardiac defects. Pediatr Cardiol 2008;29:628-36.

16. van den Berghe G, Wouters P, Weekers F, et al. Intensive insulin therapy in critically ill patients. New Engl J Med 2001;345:1359-67.

17. Van den Berghe G, Wilmer A, Hermans G, et al. Intensive insulin therapy in the medical ICU. New Engl J Med 2006;354:449-61.

18. Investigators N-SS, Finfer S, Chittock DR, et al. Intensive versus conventional glucose control in critically ill patients. New Engl J Med 2009;360:1283-97.

19. Investigators N-SS, Finfer S, Liu B, et al. Hypoglycemia and risk of death in critically ill patients. New Engl J Med 2012;367:1108-18.

20. Preiser JC, Devos P, Ruiz-Santana S, et al. A prospective randomised multi-centre controlled trial on tight glucose control by intensive insulin therapy in adult intensive care units: the Glucontrol study. Intensive Care Med 2009;35:1738-48. 


\section{Tight glycemic control in PICU}

21. Griesdale DE, de Souza RJ, van Dam RM, et al. Intensive insulin therapy and mortality among critically ill patients: a meta-analysis including NICE-SUGAR study data. CMAJ 2009;180:821-7.

22. Wiener RS, Wiener DC, Larson RJ. Benefits and risks of tight glucose control in critically ill adults: a meta-analysis. the journal of the American Medical Association. JAMA 2008;300:933-44.

23. Vlasselaers D, Milants I, Desmet L, et al. Intensive insulin therapy for patients in paediatric intensive care: a prospective, randomised controlled study. Lancet 2009;373:547-56.

24. Jeschke MG, Kulp GA, Kraft R, et al. Intensive insulin therapy in severely burned pediatric patients: a prospective randomized trial. Am J Respir Crit Care Med 2010;182:351-9.

25. Agus MS, Steil GM, Wypij D, et al. Tight glycemic control versus standard care after pediatric cardiac surgery. New Engl J Med 2012;367:1208-19.

26. Macrae D, Grieve R, Allen E, et al. A randomized trial of hyperglycemic control in pediatric intensive care. New Engl J Med 2014;370:107-18.

27. Agus MS, Wypij D, Hirshberg EL, et al. Tight glycemic control in critically ill children. New Engl J Med 2017;376:729-41.

28. Clarke M, Horton R. Bringing it all together: Lancet-Cochrane collaborate on systematic reviews. Lancet 2001;357:1728.

29. Stroup DF, Berlin JA, Morton SC, et al. Meta-analysis of observational studies in epidemiology: a proposal for reporting. Meta-analysis Of Observational Studies in Epidemiology (MOOSE) group. JAMA 2000;283:2008-12.

30. Gielen M, Mesotten D, Wouters PJ, et al. Effect of tight glucose control with insulin on the thyroid axis of critically ill children and its relation with outcome. J Clin Endocrinol Metab 2012;97:3569-76.

31. Jonassen AK, Brar BK, Mjos OD, Sack MN, Latchman DS, Yellon DM. Insulin administered at reoxygenation exerts a cardioprotective effect in myocytes by a possible anti-apoptotic mechanism. J Mol Cell Cardiol 2000;32:757-64.

32. Tune JD, Mallet RT, Downey HF. Insulin improves contractile function during moderate ischemia in canine left ventricle. Am J Physiol 1998;274: H1574-81.

33. Aljada A, Dandona P. Effect of insulin on human aortic endothelial nitric oxide synthase. Metabolism 2000;49:147-50.

34. Grover A, Padginton C, Wilson MF, Sung BH, Izzo JL Jr, Dandona P. Insulin attenuates norepinephrine-induced venoconstriction. An ultrasonographic study. Hypertension 1995;25:779-84.

35. Aljada A, Ghanim H, Saadeh R, Dandona P. Insulin inhibits NFkappaB and MCP-1 expression in human aortic endothelial cells. J Clin Endocrinol Metab 2001;86:450-3.
36. Dandona P, Aljada A, Mohanty P, et al. Insulin inhibits intranuclear nuclear factor kappaB and stimulates IkappaB in mononuclear cells in obese subjects: evidence for an anti-inflammatory effect? J Clin Endocrinol Metab 2001;86:3257-65.

37. Aljada A, Ghanim H, Mohanty P, Kapur N, Dandona P. Insulin inhibits the pro-inflammatory transcription factor early growth response gene-1 (Egr)-1 expression in mononuclear cells (MNC) and reduces plasma tissue factor (TF) and plasminogen activator inhibitor-1 (PAI-1) concentrations. J Clin Endocrinol Metab 2002;87:1419-22.

38. Malmberg K, Ryden L, Wedel H, et al. Intense metabolic control by means of insulin in patients with diabetes mellitus and acute myocardial infarction (DIGAMI 2): effects on mortality and morbidity. Eur Heart J 2005;26:650-61.

39. Hirshberg E, Lacroix J, Sward K, Willson D, Morris AH. Blood glucose control in critically ill adults and children: a survey on stated practice. Chest 2008;133:1328-35.

40. Branco RG, Xavier L, Garcia PC, et al. Prospective operationalization and feasibility of a glycemic control protocol in critically ill children. Pediatr Crit Care Med 2011;12:265-70.

41. Duvanel CB, Fawer CL, Cotting J, Hohlfeld P, Matthieu JM. Long-term effects of neonatal hypoglycemia on brain growth and psychomotor development in small-for-gestational-age preterm infants. J Pediatr 1999;134:492-8.

42. Lucas A, Morley R, Cole TJ. Adverse neurodevelopmental outcome of moderate neonatal hypoglycaemia. BMJ 1988;297:1304-8.

43. Mesotten D, Gielen M, Sterken C, et al. Neurocognitive development of children 4 years after critical illness and treatment with tight glucose control: a randomized controlled trial. JAMA 2012;308:1641-50.

44. McKinlay CJ, Alsweiler JM, Ansell JM, et al. Neonatal glycemia and neurodevelopmental outcomes at 2 years. New Engl J Med 2015;373: 1507-18.

45. McKinlay CJD, Alsweiler JM, Anstice NS, et al. Association of neonatal glycemia with neurodevelopmental outcomes at 4.5 years. JAMA Pediatr 2017;171:972-83.

46. Tasker RC. Pediatric critical care, glycemic control, and hypoglycemia: what is the real target? JAMA 2012;308:1687-8.

47. Sadhwani A, Asaro LA, Goldberg C, et al. Impact of tight glycemic control on neurodevelopmental outcomes at 1 year of age for children with congenital heart disease: a randomized controlled trial. J Pediatr 2016;174:193-8 e2. 